to the anterior communicating artery whereas the aneurysm pointed towards anterior or anteroinferior, the approach is proceeded via frontal lobe at the point of $A_{2}$ portion bifurcation.

3) Middle cerebral artery aneurysm: The approach is proceeded alongside with the main branch of middle cerebral artery by separating the sylvian fissure from fronto-temporal approach.

The temporal approach by incision of temporal lobe is introduced in case the aneurysm pointed antero-upward direction or combined with intracerebral hematoma.

4) Internal carotid artery aneurysm: The approach to internal carotid artery is achived alongside with sphenoid ridge and optic nerve via fronto-temporal approach. Various new type of clips are selected appropriately in each case.

We have 7 cases of the internal carotid artery ligation in which one postoperative death is beckoned. However Matas test for evaluating the cross circulation function is rather non-reliable for full dependency. However the carotid ligation can't be neglected for the aneurysm located below the clionoid process.

\title{
J-7. On the Surgery of the Aneurysm of the Middle Cerebral Artery
}

\author{
Tadashi AIBA \\ Department of Neurosurgery, University of Gunma
}

\section{J-8. Factors Influencing Mortality in Intracranial Aneurysm Surgery}

\author{
Fumio Nomura, Kiyoto Takao \\ Yukio Tagashira, Kimiyuki Hori and Takao Mitsuno \\ Seiya Shirakata, Shigekiyo Fujita \\ Norihiko Tamaki and Hideyuki Yamashita \\ 1st Department of Neurological Surgery, Kobe University School of Medicine
}

In the past 6 years, the cases of intracranial aneurysm treated in our clinic were 91 in total number ( 101 in the number of aneurysm), including 83 cases with a simple aneurysm and 8 cases with multiple aneurysm. There were 54 aneurysms in the internal carotid artery, 27 in the anterior communicating artery, 14 in the middle cerebral artery, 5 in the anterior cerebral artery and one in the vertebral artery.

The total number of operated cases was 73 including 69 cases treated with direct 